\title{
Li Fraumeni syndrome, cancer and senescence: a new hypothesis
}

\author{
Pan Pantziarka
}

\begin{abstract}
Li Fraumeni Syndrome (LFS) is a rare autosomal dominant hereditary cancer syndrome characterized by germline mutations in the TP53 tumour suppressor gene. Sufferers are prone to early onset cancers, particularly sarcomas, adrenocortical carcinoma and breast cancer. Cells from LFS sufferers are known to exhibit telomere dysfunction, genomic instability and spontaneous immortalisation. It is hypothesized that these facets of the LFS host are evidence that the host environment is "primed" for carcinogenesis over and above the lack of p53 tumour suppressor function. Further, it is hypothesized that the host presents an ideal environment for "two compartment tumour metabolism" to take place. Evidence from recent studies supports this new view of LFS and suggests that disrupting certain features of the host environment may markedly reduce the incidence of cancer in LFS sufferers.
\end{abstract}

Keywords: Li fraumeni syndrome, TP53, Two compartment tumour metabolism, Reverse warburg effect, Senescence, Autophagy

\section{Background}

Li Fraumeni Syndrome (LFS) is a rare autosomal dominant hereditary cancer syndrome characterized by germline mutations in the TP53 tumour suppressor gene. The syndrome is associated with a range of cancers, particularly sarcomas, gliomas, adrenocortical and breast carcinomas as well as other malignancies, particularly during childhood and early adulthood [1,2]. Among women with LFS, the most common disease is breast cancer, with a $49 \%$ risk of developing the disease by age 60 [3]. Overall the lifetime risk of cancer is estimated at $52 \%$ by age 40 years and $80 \%$ by age 50 years and for women the life-time risk has been estimated at $100 \%$ in one study $[2,4,5]$.

The "two compartment tumour metabolism" hypothesis is a new paradigm that describes a metabolic shuttle between autophagic cells in the tumour stroma and tumour cells [6,7]. The hypothesis suggests that cancer cells induce oxidative stress in the stroma by secreting hydrogen peroxide in surrounding tissues. Cancerassociated fibroblasts respond to this environmental challenge by activation and entry into an autophagic state and undergo mitophagy, mitochondrial dysfunction and a shift of metabolism towards aerobic glycolysis. This metabolic shift in cancer-associated fibroblasts

Correspondence: anticancer.org.uk@gmail.com

The George Pantziarka TP53 Trust, London, UK results in the production of high energy by-products such as 1-lactate, ketones, glutamine and other mitochondrial fuels that the tumour cells require to drive growth $[8,9]$.

At the heart of this relationship between tumour cells and the surrounding stromal tissues is the autophagic response to oxidative stress [10]. Recent evidence points to a relationship between cellular senescence and autophagy, suggesting that they are part of the same "autophagy-senescence transition (AST)", and that they both promote the anabolic growth of cancer cells. It also links aging and cancer in a radically new way, suggesting that cancer is a disease of "accelerated host aging" in the tumour stroma $[6,11]$.

\section{Presentation of the hypothesis}

In this paper we present the novel hypothesis that many of the features of the "two compartment" model of cancer, including accelerated host aging, are present in the non-cancerous LFS host, and that people with LFS are therefore "primed" for cancer development over and above a simple loss of p53 tumour suppressor function. Evidence for the hypothesis is reviewed, and the clinical implications discussed.

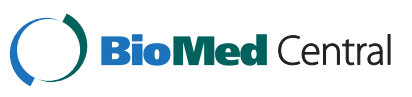

(c) 2013 Pantziarka; licensee BioMed Central Ltd. This is an Open Access article distributed under the terms of the Creative Commons Attribution License (http://creativecommons.org/licenses/by/2.0), which permits unrestricted use, distribution, and reproduction in any medium, provided the original work is properly cited. 


\section{Telomeres, senescence and immortalisation}

Telomeres are regions of nucleotide sequences that cap the ends of each chromosome and serve to protect the chromosome from recombination or degradation. Successive cell division leads to a shortening of telomere lengths, a process that can lead to chromosomal instability and which is associated both with aging and the pathology of a number of diseases, including cancer. In many respects telomere length can be seen as an indicator of biological aging independent of chronological age [12-14]. Crucially, shortened telomeres activate p53 to trigger a DNA damage response that can lead to senescence or apoptosis [15].

Clinical evidence exists that LFS patients have shorter telomeres than age-matched non-LFS individuals [16]. In other studies, children with LFS were shown to have mean telomere length shorter than unaffected parents or siblings [17]. These findings are in line with evidence from LFS fibroblast cell lines derived from patients and in p53-/- and p53+/- knockout mice [18]. In an analysis performed in 2007, shorter telomere length was associated with a younger age of cancer onset in LFS patients, and there was convincing evidence of increased telomere attrition in succeeding generations [19].

Analysis of non-malignant fibroblasts and other cells derived from LFS patients has shown that they display unusual patterns of senescence and that some of them are able to undergo spontaneous immortalisation in vitro. Where control fibroblasts from skin biopsies undergo senescence in the normal way in cultures, some of the fibroblasts from a number of LFS patients enter a long period of growth slowdown and replicative senescence during which they alter morphology, suffer chromosomal damage, including aneuploidy and telomeric association, followed by escape from senescence and the resumption of cell division and replication.

It should be noted that spontaneous immortalisation of human fibroblasts almost never occurs in cultures from non-LFS patients [20,21]. Furthermore, immortalized LFS fibroblasts are not directly tumorigenic when transplanted into nude mice. So while this immortalisation of cells is a necessary condition of malignant transformation it is not a sufficient condition [20].
Escape from senescence is often associated with the over-expression of telomerase reverse transcriptase (TERT), the enzyme complex that re-synthesizes the telomere 'caps' at the end of the chromosome. Increased expression of telomerase is common to many cancers, conversely this enzyme is absent from non-transformed cells [22]. Some tissues may be more susceptible to this process than others, as shown in the work of Shay et al. who demonstrated that breast epithelial cells immortalised more frequently than fibroblasts in cultures from an LFS patient [23]. Comparison of the telomerase staining for the different cell types showed significantly higher levels in the breast epithelial cells than in the stromal fibroblasts.

This cell-type specific difference is important in that it is a possible factor in the patterns of cancer incidence in LFS. Breast cancer in LFS affected women is the most common form of the disease, occurring in about $50 \%$ of female TP53 mutation carriers (although risk to those that survive to 50 years may be in excess of $70 \%$ ). Site specific escape from senescence might also explain the prevalence of bone and soft tissue sarcomas, adrenocortical carcinoma and other forms of cancer that are rare in the general population but common in LFS.

This differential rate of immortalisation and senescence also leads to a situation where small populations of immortalized epithelial cells, subject to mutation and chromosomal change, are surrounded by populations of cells with reduced telomere lengths and already in senescence or becoming senescent in response to oxidative stress (Figure 1). In the context of LFS and the "two compartment" model the stage is set for these immortalized epithelial cells to undergo malignant transformation and to activate the fibroblastic cells in the stroma [24].

\section{TP53, Autophagy and oxidative stress}

p53 also plays a role in cellular homeostasis, metabolism and in how cells respond to nutrient deficiency, hypoxia and other stresses. p53 can up regulate oxidative phosphorylation (OXPHOS) by inducing the synthesis of cytochrome c oxidase $(\mathrm{SCO} 2)$ and down-regulate glycolysis

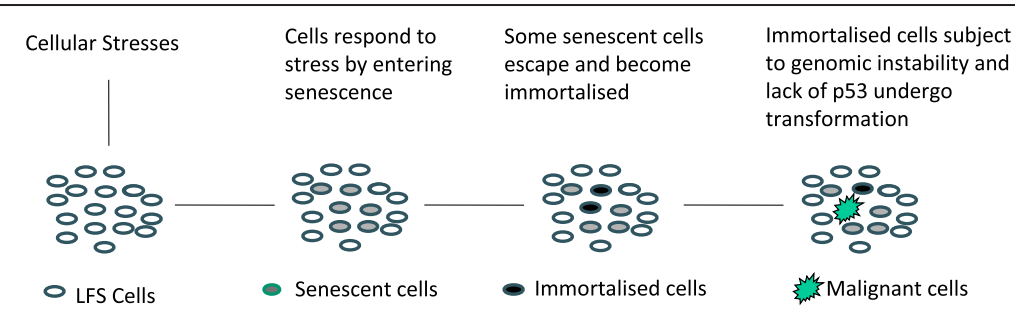

Figure 1 LFS cells with shorter telomere lengths and lacking functional p53 respond to cellular stresses, including oxidative stress, by moving into premature senescence, immortalisation and malignant transformation. 
through activation of TP53-induced glycolysis regulator (TIGAR) [25-27].

Activated cancer associated fibroblasts undergo transition to an autophagic state according to the "two compartment" hypothesis. Mechanistically, one of the drivers of this transition is an increased rate of oxidative stress due to secreted reactive oxygen species from adjacent cancer cells [28]. There is increasing evidence that p53 is an important regulator of the shift to autophagy. The picture is complex and it appears that p53 may have a dual effect on autophagy, acting as a promoter or inhibitor depending on its localization in the cell $[29,30]$. However, Tasdemir et al. have shown that inhibition, knockout or knock-down of p53 acts as a potent inducer of autophagy in a range of cell types, including fibroblasts [31]. Lisanti and colleagues specifically link this increased oxidative stress with accelerated host aging in the tumour microenvironment $[11,28]$.

Analysis of the redox parameters in blood samples of healthy LFS patients compared to non-carriers of TP53 mutations found that the LFS mutation carriers had significantly increased indicators of oxidative stress, including a four-fold increase in plasma malondialdehyde levels, indicating increased lipid peroxidation [32]. This is, therefore, further evidence of the priming of the LFS host with the pre-conditions for cancer initiation and progression according to the "two compartment" theory.

\section{The role of Cav-1}

Caveolin-1 (cav-1) is the principal structural component of caveolae, plasma membrane invaginations that participate in diverse cellular activities and are abundant in many cell types. Cav-1 regulates critical cell functions including proliferation, apoptosis, cell differentiation, and transcytosis via diverse signalling pathways. Cav-1 knock-out (KO) mice are an established animal model of premature aging, exhibiting shorter life-span, increased glucose tolerance, insulin resistance and other age-related conditions. Additionally cav-1 $\mathrm{KO}$ mice show increased oxidative stress and mitochondrial dysfunction, which are also markers of accelerated host aging [33].

A key hallmark of Lisanti's "two compartment" theory is a loss of stromal cav-1 and a corresponding upregulation of cav-1 in tumour cells (i.e. the effects of cav-1 are compartment-specific) [34]. Loss of stromal cav-1 expression is a key indicator of the effect of immortalized epithelial cells on adjacent fibroblasts $[35,36]$.

A recent study by Sherif and Sultan analysed cav-1 expression in non-cancerous LFS fibroblasts and reported that affected family members showed an $88 \%$ downregulation of cav-1 compared to non-affected family members [37]. This new finding is directly as predicted by our hypothesis and confirms that another key feature of the "two compartment" model, (and one that is also related to characteristics of accelerated host aging), is present in the non-malignant LFS host environment.

\section{Summary}

We have outlined a number of important characteristics of the "two compartment" model and have shown how these exist in the non-cancerous LFS host, as shown in Table 1. Furthermore, we have presented evidence that these characteristics are closely associated with the accelerated host aging described in the "two compartment" model of cancer.

\section{Testing the hypothesis}

Our hypothesis predicts that disrupting the "priming" of the host environment will reduce cancer initiation and progression in LFS sufferers and can be tested by undertaking specific interventions. Specifically, there are three possible targets:

- inhibiting senescence in stromal cells

- inducing autophagy in malignant cells/inhibiting autophagy in stromal cells

- interrupting the metabolic shuttle between stromal fibroblasts and tumour cells

These three targets alter the host environment in such a way as to disrupt the "priming" towards the two compartment model, and in theory would markedly reduce the risk of developing cancer in LFS sufferers. A number of important results already suggest that these mechanisms can make a significant impact on cancer risk for LFS sufferers.

Komarova and colleagues showed that the mTOR inhibitor rapamycin, which is known to inhibit cellular senescence, increased lifespan and decreased the incidence of spontaneous tumours in p53 +/- mice [38]. The effect was stronger when started early in life, suggestive of a systemic effect in the host rather than in direct anti-tumour activity. According to our hypothesis rapamycin will decrease the number of cells which enter senescence and hence reduce the number which escape from this state and achieve immortalisation, in turn

Table 1 Characteristics of accelerated host aging in tumours and in the non-cancerous LFS host

\begin{tabular}{lcc}
\hline Characteristic & $\begin{array}{c}\text { Two compartment } \\
\text { tumour model }\end{array}$ & $\begin{array}{c}\text { Non-cancerous } \\
\text { LFS host }\end{array}$ \\
\hline Senescent Fibroblasts & $\checkmark$ & $\checkmark$ \\
High Oxidative Stress & $\checkmark$ & $\checkmark$ \\
Loss of Stromal Cav-1 & $\checkmark$ & $\checkmark$ \\
Tumour Cells Resistant & $\checkmark$ & $\checkmark$ \\
to Autophagy & & \\
\hline
\end{tabular}


reducing the number of cells liable to undergo malignant transformation. In other words, decreasing senescence will interrupt the progression shown in Figure 1. Similarly, inhibiting senescence will also stop stromal cells responding to the oxidative stress produced by established tumours by moving into an autophagic state wherein they can "feed" high-energy food to the coupled tumour cells. Thus, retarding the onset of senescence has compartment-specific effects which interrupt the metabolic shuttle between tumour and stroma.

While inhibiting senescence is one possible means of interrupting this metabolic shuttle, another possible mechanism is to induce autophagy in the tumour compartment. The "two compartment" model shows that activated stromal fibroblasts respond to the oxidative stress generated by tumours by becoming autophagic and switching metabolism to aerobic glycolysis, which generates high-energy fuels that can drive the growth of tumour cells $[6,8]$. However, inducing autophagy in the tumour compartment disrupts the metabolic shuttle as the tumour cells, depending on mitochondrial oxidative phosphorylation, are no longer able to feed on the supply of nutrients from the stroma. Alternatively, inhibiting autophagy in the stroma will deliver the same outcome. One possible mechanism to achieve this is through the use of the autophagy-inhibitor chloroquine, which is currently being used in a number of clinical trials in combination with a range of chemotherapeutic agents.

Finally, the hypothesis suggests that carcinogenesis and disease progression in LFS is sensitive to the metabolic state of cancer associated fibroblasts and adjacent tumour cells. Mutant p53 is stabilised by increased glucose supply, and high levels of mutant p53 act as an inhibitor of autophagy in cancer cells [39]. Reducing glucose supply, therefore, will reduce the stabilisation of mutant p53. It will also decrease the nutrient supply to the stromal fibroblasts which in turn reduces the supply of fuels to "feed" the tumour cells.

Another prediction of the hypothesis is, therefore, that cancer incidence in LFS can be reduced by restriction of the supply of glucose. Options for altering the availability of glucose include dietetic alterations or pharmacological interventions. Supporting evidence for a metabolic influence in LFS carcinogenesis is provided by work on p53 +/- mice, which showed that calorie restriction in adult animals delayed the development of cancer [40].

Chief among the pharmacological interventions is the use of the anti-diabetic drug metformin, already in clinical trials in combination with standard chemotherapy agents. Metformin is known to target many of the pathways affected by dietary caloric restriction, including AMPK, mTOR and IGFR [41]. In the context of the LFS phenotype it would have dual effects. First it acts to restrict the supply of glucose, through the inhibition of hepatic glucose production, to activated stromal cells. Secondly it can act to block mitochondrial oxidative phosphorylation in the tumour cells, hence acting to starve cancer cells through two distinct pathways. There is also some evidence that metformin can selectively induce apoptosis in p53-deficient cells under-going nutrient stress, which is of significant interest [42].

\section{Implications of the hypothesis}

In LFS cancer initiation is more likely than in the general population and may be triggered by the genetic instability that results from shortened telomere lengths, which may be exacerbated by high basal levels of oxidative stress, and by a lack of functional p53. Once cancer is initiated the host environment is already in a state where stromal fibroblasts respond to tumour cells by becoming activated and moving into a state of autophagy, mitophagy and switching metabolism to aerobic glycolysis, thereby feeding the tumour cells with the high energy by-products of this form of metabolism. In short, cancer in LFS patients rapidly moves to a state of "two compartment" tumour metabolism.

To date the common understanding has been that LFS patients are at greater risk of developing malignancies because of the accumulation of secondary mutations over and above the mutated TP53. However, the "two compartment tumour metabolism" hypothesis and the additional data outlined above suggest that p53 loss in the stroma accelerates the process of their recruitment by immortalized epithelial cells to promote tumour formation. Thus malignancy in LFS is associated with cellular senescence in stromal cells in response to increased oxidative stress from epithelial cells, a process which may be regarded as a form of accelerated host aging [11].

The hypothesis outlined here is consistent with the patterns of cancer incidence in LFS affected families, for example, the tendency to early onset cancers of specific tissues, such as breast cancer or soft tissue or bone sarcomas, rather than an across the board tendency to all forms of cancer. In particular, it may shed light on the paradox that LFS patients do not show an increased incidence of cancers related to tobacco, environmental toxins or occupation, as would be expected if damaged DNA repair mechanisms were the primary outcome from loss of TP53 function [43]. It may also explain the higher incidence of cancer before the age of 40 , as those individuals with the "more aged" LFS phenotype (shorter telomeres, fibroblastic cells more likely to move into senescence and autophagy, higher levels of basal oxidative stress, greater loss of cav-1) develop cancer at a younger age than those with a more normal telomeric profile (a "less aged" phenotype).

This shift in emphasis from a focus on accumulated DNA damage to cell-type interactions and accelerated 
aging has important therapeutic implications for LFS. Currently there are no cancer prevention strategies in place for LFS sufferers. Newly diagnosed patients are subject to varying levels of surveillance but are offered no practical steps to reduce the risk of developing malignancies other than bilateral risk reducing mastectomy in women. This imposes high levels of stress on patients and their families, particularly for parents of LFS children and in families with extensive cancer histories.

The hypothesis outlined here opens the door to active chemo-preventative strategies in terms of autophagy inhibition, steps to reduce oxidative stress and so on. Drugs such as the anti-diabetic drug metformin, the autophagy inhibitor chloroquine and other agents with low toxicity, including anti-oxidants, may also be worthy of further investigation in LFS families.

Research is also warranted to ascertain whether shorter telomere length, reduced cav-1 expression or increased oxidative stress have prognostic significance in LFS.

\section{Competing interests}

The author declares that he has no competing interests.

\section{Acknowledgements}

Publication was funded by the George Pantziarka TP53 Trust. Thanks are due to Anthony Howell, Gareth D. Evans and Michael Lisanti for support and encouragement.

Received: 8 February 2013 Accepted: 13 April 2013

Published: 15 April 2013

\section{References}

1. Nichols KE, Malkin D, Garber JE, Fraumeni JF Jr, Li FP: Germ-line p53 mutations predispose to a wide spectrum of early-onset cancers. Cancer Epidemiol Biomarkers Prev 2001, 10:83-87.

2. Malkin D: Li-Fraumeni Syndrome Genes and. Cancer 2011, 2(4):475-484.

3. Masciari S, Dillon DA, Rath M, Robson M, Weitzel JN, Balmana J, et al: Breast cancer phenotype in women with TP53 germline mutations: a LiFraumeni syndrome consortium effort. Breast Cancer Res Treat 2012, 133(3):1125-1130

4. Chompret A, Brugières L, Ronsin M, Gardes M, Dessarps-Freichey F, Abel A, et al: P53 germline mutations in childhood cancers and cancer risk for carrier individuals. Br J Cancer 2000, 82(12):1932-1937.

5. Wu CC, Shete S, Amos Cl, Strong LC: Joint effects of germ-line p53 mutation and sex on cancer risk in Li-Fraumeni syndrome. Cancer Res 2006, 66(16):8287-8292.

6. Salem AF, Whitaker-Menezes D, Lin Z, Tanowitz HB, Al-Zoubi MS, Howell A, et al: Two-compartment tumor metabolism: Autophagy in the tumor microenvironment, and oxidative mitochondrial metabolism (OXPHOS) in cancer cells. Cell Cycle 2012, 11(13):2545-2556.

7. Carito V, Bonuccelli G, Martinez-Outschhoorn UE, Whitaker-Menezes D, Caroleo MC, Cione E, et al: Metabolic remodeling of the tumor microenvironment: Migration stimulating factor ( $\mathrm{ms}$ ) reprograms myofibroblasts towards lactate production, fueling anabolic tumor growth. Cell Cycle 2012, 11(18):3403-3414.

8. Bonuccelli G, Tsirigos A, Whitaker-Menezes D, Pavlides S, Pestell RG, Chiavarina B, et al: Ketones and lactate "fuel" tumor growth and metastasis: Evidence that epithelial cancer cells use oxidative mitochondrial metabolism. Cell Cycle 2010, 9(17):3506-3514.

9. Fiaschi T, Marini A, Giannoni E, Taddei ML, Gandellini P, De Donatis A, et al: Reciprocal Metabolic Reprogramming Through Lactate Shuttle Coordinately Influences Tumor-Stroma Interplay. Cancer Res 2012, 72(19):5130-5140.

10. Pavlides S, Tsirigos A, Migneco G, Whitaker-Menezes D, Chiavarina B, Flomenberg $\mathrm{N}$, et al: The autophagic tumor stroma model of cancer: Role of oxidative stress and ketone production in fueling tumor cell metabolism. Cell Cycle 2010, 9:3485-3505.

11. Lisanti MP, Martinez-Outschoorn UE, Pavlides S, Whitaker-Menezes D, Pestell RG, Howell A, Sotgia F: Accelerated aging in the tumor microenvironment: connecting aging, inflammation and cancer metabolism with personalized medicine. Cell Cycle 2011, 10(13):2059-2063.

12. Fyhrquist F, Saijonmaa O: Telomere length and cardiovascular aging. Ann Med 2012, 44(Suppl 1):S138-S142.

13. Blasco MA: Telomeres and human disease: ageing, cancer and beyond. Nat Rev Genet 2005, 6(8):611-622.

14. Aubert G, Lansdorp PM: Telomeres and aging. Physiol Rev 2008, 88(2):557-579.

15. Deng $Y$, Chang $S$ : Role of telomeres and telomerase in genomic instability, senescence and cancer. Lab Invest 2007, 87(11):1071-1076.

16. Trkova M, Prochazkova K, Krutilkova V, Sumerauer D, Sedlacek Z: Telomere length in peripheral blood cells of germline TP53 mutation carriers is shorter than that of normal individuals of corresponding age. Cancer 2007, 110(3):694-702.

17. Tabori U, Nanda S, Druker H, Lees J, Malkin D: Younger Age of Cancer Initiation Is Associated with ShorterTelomere Length in Li-Fraumeni. Syndrome Cancer Res 2007, 67(4):1415-1418.

18. Kruk PA, Bohr VA: Telomeric length in individuals and cell lines with altered p53 status. Radiat Oncol Investig 1999, 7(1):13-21.

19. Tabori U, Nanda S, Druker H, Lees J, Malkin D: Younger age of cancer initiation is associated with shorter telomere length in Li-Fraumeni syndrome. Cancer Res 2007, 67(4):1415-1418.

20. Bischoff FZ, Yim SO, Pathak S, Grant G, Siciliano MJ, Giovanella BC, et al: Spontaneous abnormalities in normal fibroblasts from patients with Li-Fraumeni cancer syndrome: aneuploidy and immortalization. Cancer Res 1990, 50(24):7979-7984.

21. Rogan EM, Bryan TM, Hukku B, Maclean K, Chang AC, Moy EL, et al: Alterations in p53 and p16INK4 expression and telomere length during spontaneous immortalization of Li-Fraumeni syndrome fibroblasts. Mol Cell Biol 1995, 15(9):4745-4753.

22. Artandi SE, Attardi LD: Pathways connecting telomeres and p53 in senescence, apoptosis, and cancer. Biochem Biophys Res Commun 2005, 331(3):881-890.

23. Shay JW, Tomlinson G, Piatyszek MA, Gollahon LS: Spontaneous in vitro immortalization of breast epithelial cells from a patient with Li-Fraumeni syndrome. Mol Cell Biol 1995, 15(1):425-432

24. Pavlides S, Whitaker-Menezes D, Castello-Cros R, Flomenberg N, Witkiewicz AK, Frank PG: The reverse Warburg effect: aerobic glycolysis in cancer associated fibroblasts and the tumor stroma. Cell Cycle 2009, 8(23):3984-4001.

25. Zhang XD, Qin ZH, Wang J: The role of $\mathrm{p} 53$ in cell metabolism. Acta Pharmacol Sin 2010, 31(9):1208-1212.

26. Bensaad K, Tsuruta A, Selak MA, Vidal MN, Nakano K, Bartrons R, et al: TIGAR, a p53-Inducible Regulator of Glycolysis and Apoptosis. Cell 2006, 126(1):107-120.

27. Reinhardt HC, Schumacher B: The p53 network: cellular and systemic DNA damage responses in aging and cancer. Trends Genet 2012, 28(3):128-136.

28. Guido C, Whitaker-Menezes D, Lin Z, Pestell RG, Howell A, Zimmers TA, et al: Mitochondrial fission induces glycolytic reprogramming in cancerassociated myofibroblasts, driving stromal lactate production, and early tumor growth. Oncotarget 2012, 3(8):798-810.

29. Levine B, Abrams J: p53: The Janus of autophagy? Nat Cell Biol 2008, 10(6):637-639.

30. Tasdemir E, Maiuri MC, Galluzzi L, Vitale I, Djavaheri-Mergny M, D'Amelio M, et al: Regulation of autophagy by cytoplasmic p53. Nat Cell Biol 2008, 10(6):676-687.

31. Tasdemir E, Maiuri MC, Orhon I, Kepp O, Morselli E, Criollo A, et al: p53 represses autophagy in a cell cycle-dependent fashion. Cell Cycle 2008, 7(19):3006-3011.

32. Macedo GS, Lisbôa Da Motta L, Giacomazzi J, Netto CB, Manfredini VS, Vanzin $C$, et al: Increased Oxidative Damage in Carriers of the Germline TP53 p.R337H Mutation. PLoS One 2012, 7(10):e47010.

33. Sotgia F, Martinez-Outschoorn UE, Pavlides S, Howell A, Pestell RG, Lisanti MP: Understanding the Warburg effect and the prognostic value of stromal caveolin-1 as a marker of a lethal tumor microenvironment. Breast Cancer Res 2011, 13(4):213.

34. Mercier I, Camacho J, Titchen K, Gonzales DM, Quann K, Bryant KG, et al: Caveolin-1 and accelerated host aging in the breast tumor microenvironment: chemoprevention with rapamycin, an mTOR inhibitor and anti-aging drug. Am J Pathol 2012, 181(1):278-293. 
35. Lee SW, Reimer CL, Oh P, Campbell DB, Schnitzer JE: Tumor cell growth inhibition by caveolin re-expression in human breast cancer cells. Oncogene 1998, 16(11):1391-1397.

36. Williams TM, Lee $H$, Cheung MW, Cohen AW, Razani B, lyengar $P$, et al: Combined loss of INK4a and caveolin-1 synergistically enhances cell proliferation and oncogene-induced tumorigenesis: role of INK4a/ CAV-1 in mammary epithelial cell hyperplasia. J Biol Chem 2004, 279(23):24745-24756.

37. Sherif ZA, Sultan AS: Divergent control of Cav-1 expression in noncancerous Li-Fraumeni syndrome and human cancer cell lines. Cancer Biol Ther 2012, 14:1.

38. Komarova EA, Antoch MP, Novototskaya LR, Chernova OB, Paszkiewicz G, Leontieva OV, et al: Rapamycin extends lifespan and delays tumorigenesis in heterozygous p53+/- mice. Aging (Albany NY) 2012, 4(10):709-714

39. Rodriguez OC, Choudhury S, Kolukula V, Vietsch EE, Catania J, Preet A, et al: Dietary downregulation of mutant p53 levels via glucose restriction. Cell Cycle 2012, 11(23):4436-4446.

40. Berrigan D, Perkins SN, Haines DC, Hursting SD: Adult-onset calorie restriction and fasting delay spontaneous tumorigenesis in p53-deficient mice. Carcinogenesis 2002, 23(5):817-822.

41. Pierotti MA, Berrino F, Gariboldi M, Melani C, Mogavero A, Negri T, et al: Targeting metabolism for cancer treatment and prevention: metformin, an old drug with multi-faceted effects. Oncogene 2013, 32:1475-1487.

42. Buzzai M, Jones RG, Amaravadi RK, Lum JJ, DeBerardinis RJ, Zhao F, et al: Systemic treatment with the antidiabetic drug metformin selectively impairs p53-deficient tumor cell growth. Cancer Res 2007, 67(14):6745-52.

43. Palmero El, Achatz MI, Ashton-Prolla P, Olivier M, Hainaut P: Tumor protein 53 mutations and inherited cancer: beyond Li-Fraumeni syndrome. Curr Opin Oncol 2010, 22(1):64-9.

doi:10.1186/1475-2867-13-35

Cite this article as: Pantziarka: Li Fraumeni syndrome, cancer and senescence: a new hypothesis. Cancer Cell International 2013 13:35.

\section{Submit your next manuscript to BioMed Central and take full advantage of:}

- Convenient online submission

- Thorough peer review

- No space constraints or color figure charges

- Immediate publication on acceptance

- Inclusion in PubMed, CAS, Scopus and Google Scholar

- Research which is freely available for redistribution 